\title{
Modeling Bus Priority Using Intermodal Dynamic Network Assignment-Simulation Methodology
}

\author{
Khaled F. Abdelghany, Southern Methodist University \\ Ahmed F. Abdelghany, Embry-Riddle Aeronautical University \\ Hani S. Mahmassani, University of Maryland \\ Akmal S. Abdelfatah, American University of Sharjah
}

\begin{abstract}
This article presents a modeling framework that represents bus priority at signalized intersections in the context of its potential network-level and intermodal effects. The model incorporates bus priority within an intermodal dynamic traffic assignmentsimulation model. It dynamically assigns travelers to different modes and routes in the network according to prevailing traffic conditions, which result from applying a certain network control/bus priority scheme. The model considers changes in traffic conditions as a result of (1) drivers' route choice adjustments due to changes in traffic signals settings and (2) modal shifts by travelers to take advantage of improved transit service. Three different bus priority strategies are considered: phase (green) extension, red truncation, and phase advance. A set of simulation experiments is performed to compare these strategies using two different assignment scenarios: single-mode assignment and intermodal assignment. The results of these experiments highlight the importance of considering reassignment and potential modal shifts in evaluating traffic network performance under different control schemes, especially
\end{abstract}


when these schemes are expected to affect the modal split in the network such as bus priority.

\section{Introduction}

An important operational goal for transit agencies is to maintain fast and reliable transit service for customers and to be competitive against private-car use. Over the last two decades, bus priority at signalized intersections has gained attention as a possible technique to improve transit vehicle travel time. Advances in detection devices and communication technologies as well as in signal controllers have enabled a variety of intelligent bus priority strategies. Provision of bus priority at traffic signals is a commonly available functionality of modern signal controllers. Several programs using different logic and strategies are available for implementation in conjunction with both isolated and networked controllers (Baker et al. 2002; Smith et al. 2005). Most real-time adaptive control systems for signalized networks provide priority capabilities for buses and other special vehicles (e.g., Emergency Management Vehicles) (Lowrie 1982; Sims and Finlay 1984; Longfoot 1982; Yagar 1993; Sunkari et al. 1995; Chang et al. 2003; Mirchandani and Lucas 2004). Three main bus priority strategies are widely applied: phase extension, red truncation, and phase advance. In the phase extension strategy, when a transit vehicle is detected and the phase that serves this vehicle is active, the green is extended to ensure that this vehicle crosses the intersection. If a transit vehicle is detected while the phase that serves it is not active, the vehicle waits until its phase is reactivated in the next cycle. Red truncation and phase advance strategies provide additional priority options for transit vehicles. They advance the green for the detected transit vehicles either immediately (phase advance) or after providing minimum or some reduced green (red truncation) to the other phases. The net effect is to reduce the stopping time of the transit vehicles at the intersection.

Several researchers have investigated the relative desirability, operational performance, and evaluation of bus priority strategies (Heydecker 1983; Chard and Lines 1987; Radwan and Benevell 1983; Bell 1992; Chang et al. 1995; Khasnabis et al. 1996; Garrow and Machemehl 1997; Baker et al. 2002; Chang and Ziliaskopoulos 2003; Nash 2003; Smith et al. 2005). Previous studies to evaluate different bus priority strategies have been limited in one or more of the following aspects:

- Studies and models that have considered bus priority at the network level have ignored the vehicles reassignment phenomenon that could accompany 
the change in a signal timing plan. A change in signal timing due to bus priority would affect travel times along several paths in the network, resulting in some switching to shorter paths by auto drivers. While this reassignment has been well recognized in the literature for signal timing in general (Abdelfatah and Mahmassani 1998), it has not been considered explicitly with bus priority. Evaluating bus priority in a dynamic assignment framework, which considers the vehicles reassignment phenomenon, provides a better evaluation of the long-term impacts of implemented strategies.

- The effect of bus priority on mode choice is essential to evaluate bus priority strategies comprehensively, and does not appear to be reported in the literature. Changes in auto and transit travel times due to transit priority may induce some tripmakers to shift from one mode to another. This would affect the total number of vehicles in the network and consequently change the overall network performance. Ignoring this dimension in previous studies will likely understate the benefits of bus priority.

The model described in this article incorporates bus priority at signalized intersections in the context of its potential intermodal network-level effects. The model considers changes in traffic conditions as a result of (1) drivers' route choice adjustments due to changes in traffic signals settings and (2) modal shifts by travelers to take advantage of improved transit service. The methodology overcomes the key limitations of previous approaches.

The intermodal assignment-simulation model (Abdelghany and Mahmassani 2001; Abdelghany 2001) represents special-purpose enhancements of the DYNASMART simulation-assignment tool developed to evaluate ITS applications in traffic networks (Jayakrishnan et al. 1994; Abdelghany et al. 1999; and Mahmassani et al. 2000). The model dynamically assigns travelers to the different modes and routes in the network according to prevailing traffic conditions, which result from applying a certain network control scheme. Two different traffic assignment scenarios are considered: single-mode assignment and intermodal assignment. In the single-mode assignment scenario, all travelers are assumed to use private cars. Transit vehicles are simulated only as background traffic for the auto traffic. This scenario examines the impact of priority primarily on network traffic conditions in situations where transit mode usage is very low, and only minimal shifts can be expected from the bus improvements. The intermodal assignment scenario considers possible change in the mode share because of change in transit vehicle travel time due to bus priority at selected signalized intersections in the network. 
Three bus priority strategies are considered in the analysis: phase extension, red truncation, and phase advance.

This article begins with a description of the assignment-simulation methodology, followed by a description of the bus priority strategies considered in the study. Different sets of experiments are then presented to show the model capabilities and to illustrate the significance of evaluating bus priority in an intermodal dynamic network assignment framework. The results of these experiments together with analysis of the main findings are provided. Conclusions and possible extensions are given in the final section.

\section{Dynamic Assignment-Simulation Methodology}

The methodology is based on the DYNASMART assignment-simulation model, enhanced for intermodal transportation network applications. The logic of the core simulation-assignment procedures is described elsewhere (Jayakrishnan et al. 1994; Abdelghany et al. 1999; Mahmassani et al. 2000), and only aspects directly relevant to the present application will be highlighted. The model considers different travel modes such as passenger cars, buses, metro/subway and highoccupancy vehicles (HOV). It captures the interaction between mode choice and traffic assignment under different traffic control schemes, and under different information provision strategies. Figure 1 illustrates the framework and the different components of the methodology.

The model generates travelers based on predetermined time-dependent origindestination (OD) zonal demands. Each generated traveler is assigned a set of attributes, which include his or her trip starting time, generation link, final destination, and a distinct identification number. An indicator is also assigned to each traveler to denote car ownership status. In parallel, transit vehicles are generated according to a predetermined schedule and follow predetermined routes. Prevailing travel times on each link are estimated using a vehicle simulation component that moves vehicles and captures the interaction between private cars and transit vehicles. The model also considers values of other attributes (e.g., parking costs, highway tolls, transit fares, out-of-vehicle time, and number of transfers along the route) that may be used by travelers to evaluate different mode-route options.

Using these attributes, a mode-route decision module is activated to provide travelers with a superior set of mode-route options. This route-mode decision module consists of a multiobjective shortest path algorithm, which generates a set of supe- 


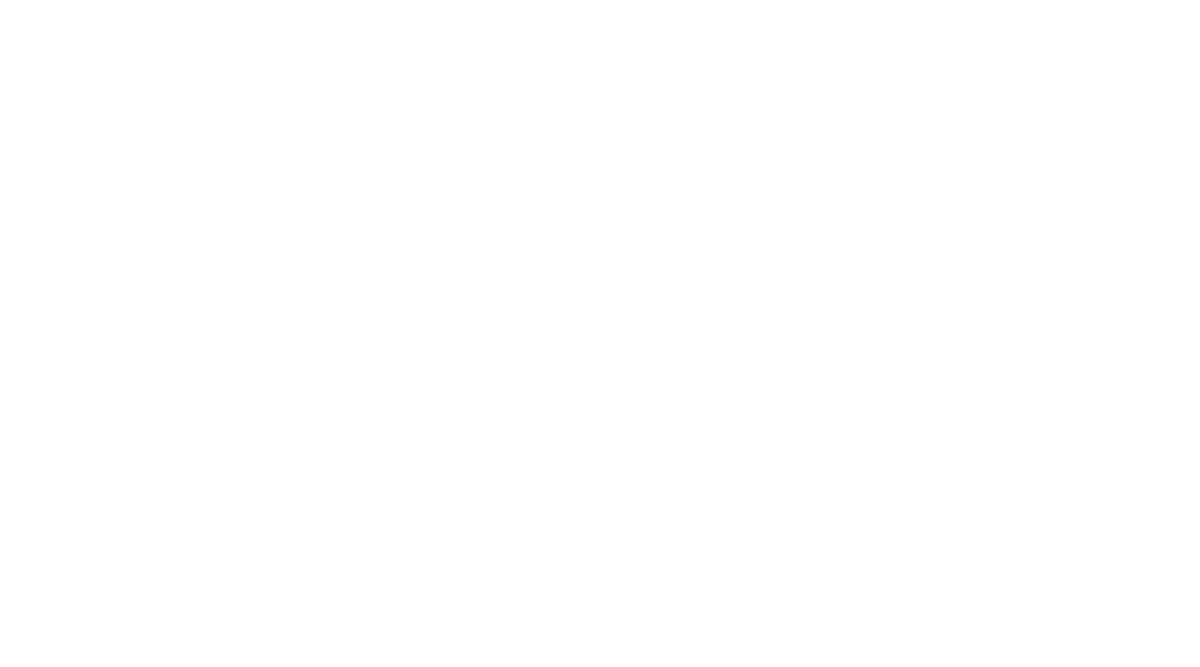

\section{Figure 1. Overall Modeling Framework}

rior paths in terms of the set (or a suitable subset) of the attributes listed above. Considering the diverse set of traveler behavioral rules as well as different levels of information availability, travelers evaluate the different mode-route options to select their preferred alternative. These behavior rules and response mechanisms are implemented through the user behavior component of the methodology. Each option represents an initial plan that a traveler follows (unless he or she receives en-route real-time information suggesting a better plan) to reach his or her final destination. This plan describes the used mode(s) and the route to be followed including any transfer node(s) along this route. Based on the available options, a traveler may choose pure mode or a combination of modes to reach his or her final destination.

If a traveler chooses private car for the whole trip or part of it, a car is generated and moved into the network with a starting time equal to its driver's starting time. Each newly generated vehicle is assigned a unique ID number. Vehicles are then moved in the network subject to the prevailing traffic conditions until they reach their final destinations or the next transfer node along the prespecified route (in the case of an intermodal trip). If a traveler chooses a transit mode, he or she is assigned to a transit line such that the passenger's destination is a node along the path followed by the bus. If no single line is found or if the passenger is not satisfied with the available single line, the passenger is assigned to a path composed of two lines with one transfer node, such that the passenger's destination is a node along 
the path followed by the second bus. If no two such lines are found, the search is continued for three lines with two transfers. It is assumed that no passenger would be willing to incur more than two transfers in his or her trip. Thus, if no path with a maximum of two transfers is available, the trip is indicated as infeasible. Given the passenger's origin node, the nearest transit stop along the first line in the passenger's path is determined, and he or she waits until the arrival of the next vehicle that serves that transit line. When a transit vehicle arrives at a certain stop, all passengers who are waiting for a vehicle serving this specific transit line get on board. These passengers depart to reach their final destination or the next transfer node along their route.

Upon the arrival of a vehicle (private car or transit vehicle) to a certain destination node, this destination is compared to the final destinations and transfer of each traveler on board. If it matches the final destination of a traveler, the current time is recorded for this traveler as his or her arrival time. If it matches a transfer, the traveler transfers to the next transit line in his or her plan. The nearest stop is again determined and the traveler waits for his or her next transit vehicle. The time difference between arrival at the transfer node and boarding of the next line is calculated as the waiting time at the current transfer node for this traveler. This process is continued until all vehicles reach their final destinations carrying all travelers. If a traveler misses the initially assigned transit vehicle because of late arrival or because the vehicle does not have enough space, the model allows the traveler to replan the trip. Available options are regenerated for this traveler and he or she makes a selection through the behavior component.

The vehicle simulation component is time-based and moves individual vehicles along links according to local speeds determined consistently with macroscopic traffic stream models (i.e., a speed-density relation of modified Greenshield's form is used in this implementation). For every time step, the number of vehicles on each link is calculated using conservation principles; numbers in each class of vehicles in the traffic mix are kept separately. Consistently with the macroscopic logic for modeling vehicle interactions, average passenger car equivalent factors are used to convert each vehicle type to the equivalent passenger car units. Also, the effect of bus stopping at a bus stop on the link is considered by reducing the link capacity for a period equal to the bus dwell time. The resulting equivalent-car concentration is then calculated for each link, and used to estimate the corresponding speed through the speed-density relation. These speeds, updated continually to reflect prevailing conditions, determine vehicular movement on that link. 
Queuing and turning maneuvers at junctions are explicitly modeled, thereby ensuring adherence to first-in, first-out principles as well as traffic control devices at junctions. Vehicles that reach the end of the link and are unable to move to a downstream link because of capacity limitations join the back of the vehicle queue at the upstream end of the link. The physical size of the queue is explicitly represented in the simulation, resulting in the division of the link into a moving part and a queuing part. Vehicles that reach the back of the queue must wait until vehicles ahead of them are discharged. All inflow and outflow constraints that limit the number of vehicles entering and leaving each link under the prevailing traffic control are implemented. The right-of-way among competing movements is allocated according to the existing control device at every intersection. The outflow constraints limit the maximum number of vehicles allowed to leave any given approach of an intersection, reflecting the available vehicles in queue and outflow capacities of the approach under the prevailing control. The inflow constraints bound the total number of vehicles that are allowed to enter a link. These constraints bound the total number of vehicles from all approaches that can be accepted by the receiving link, which reflects both physical storage consideration and inflow throughput capacity.

\section{Signal Control and Bus Priority Strategies}

The DYNASMART model and its intermodal extension provide the ability to explicitly model an array of control devices for street intersections such as yield signs, stop signs and signal control, which includes pretimed and actuated control. In this article, three bus priority strategies are evaluated. This section describes the simulation logic of the actuated signal logic and the bus priority algorithm. A description of the logic of the other control elements can be found in $\mathrm{Hu}$ (1995).

Figures 2, 3, and 4 illustrate the actuation logic for the three different bus priority strategies, namely phase extension, red truncation, and phase advance, respectively. These strategies are implemented by modifying the vehicle-actuated signal control logic of DYNASMART. In this logic, the green time for a given phase is determined based on the number of vehicles that would have reached the intersection at the end of the current simulation interval (in the absence of a queue). The green time is subsequently extended as appropriate at each simulation interval until "max out" is reached, or terminated if no longer needed, thereby emulating "gap out." 
Figure 2. Logic of Actuated Signal Control with Phase Extension Bus Priority 


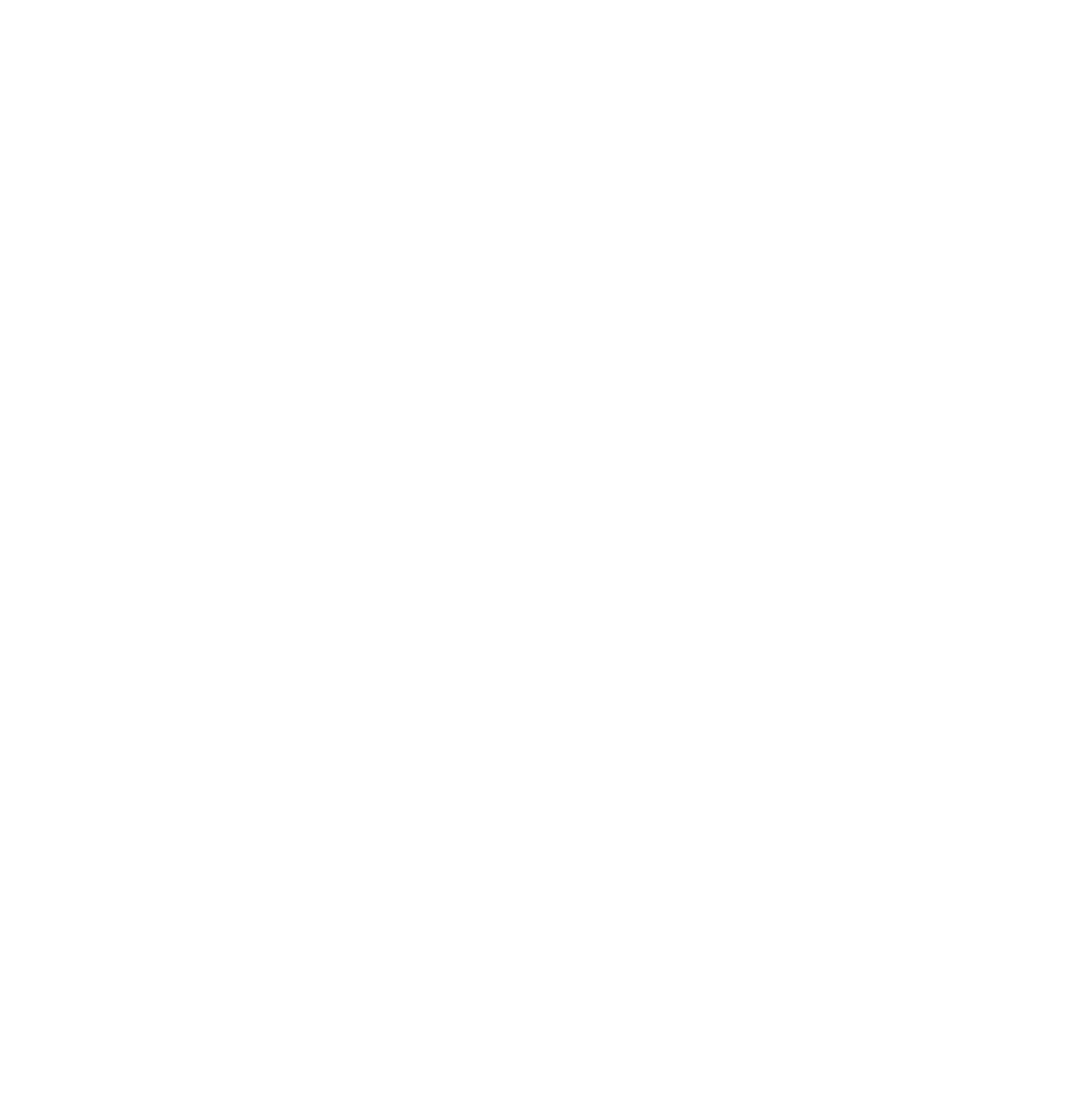

Figure 3. Logic of Actuated Signal Control with Red Truncation Bus Priority

In the phase extension case, if a bus is detected on any of the approaches served during the current phase, the green time required to move this bus through the signal is estimated. If the extension required for the bus is greater than the extension required to accommodate all vehicles that would have reached the stop line over the red interval, the green time for this phase is extended unless the maximum green value is reached. In other words, the green extension should be within the allowable maximum green time for that phase, and no exception is made for the bus. The logic of this priority strategy is detailed in Figure 2. Assume the start 


\section{Figure 4. Logic of Actuated Signal Control with Phase Advance Bus Priority}

time of phase $i$ is $t_{i, s}$. The current green time of phase $i$ is $G_{i, T-1}$, which is assigned to phase $i$ at the end of simulation interval $T-1$. For each simulation interval, the green time extension, if needed, is calculated as the longer of the time required to accommodate all vehicles that would reach the stop line at the end of the simulation time interval $T$ and the time required to free any detected bus on any of the approaches that are served during phase $i$. If the remaining green time, $G_{i, T-1}-(t$ $\left.-t_{i, s}\right)$, is less than this required extension, the calculated green extension is added to the green time of the phase $i$. If the allocated time exceeds the maximum green time, the maximum green time is given to this phase. Otherwise, the signal enters 
the change interval and switches to another phase. Thus, the next phase $j$ starts at time $t_{j, s}=G_{i, T}+t_{i, s}+$ yellow $_{i}$ and has the green time $G_{j, T}=G_{\text {min }}$.

In the red truncation and phase advance cases, if a bus is detected on any of the intersection approaches, one of the following two cases could be encountered. First, the phase that serves this bus is already active. In this case, the phase extension logic described above is followed and no change in the phase sequence is made. Second, the phase that serves the detected bus is not the active phase. In this case, the phase that serves the detected bus is somehow advanced to minimize bus delay at the intersection.

If the red truncation logic is implemented, only the minimum green value plus the yellow interval is given to the remaining phases in the cycle to advance the bus phase. For example, consider Figure 3, which represents the three-phase traffic signal in the sequence of $i, j$, and $k$, respectively. If a bus is detected in one of the approaches that is served by phase $i$, while phase $j$ is active, both phases $j$ and $k$ are given the minimum green plus yellow. Then, phase $i$ is initiated to clear the bus.

If the phase advance is implemented, the green of the active phase is immediately cut off (after providing the appropriate yellow interval) and the bus phase is activated directly. For example, in Figure 4, if a bus is detected in one of the approaches that is served by phase $i$, while phase $j$ is active, the green is cut in phase $j$ and a yellow is given. Also, phase $k$ is completely skipped to start phase $i$ to clear the bus.

\section{Experimental Design}

A set of simulation experiments are designed to illustrate the model capabilities. Figure 5 depicts the test network used in these experiments, which represents the south-central corridor in Fort Worth, Texas. The network consists of part of about $22 \mathrm{~km}$ of the freeway (I-35W) surrounded by a street network with a total of 178 nodes and 441 links. All signalized intersections (61 intersections) are assumed to have vehicle-actuated controls and capable of implementing the three bus priority strategies described earlier. The maximum green value was set as 25 seconds for the four-phase intersections and 55 seconds for the two-phase intersections. The network does not contain three-phase signalized intersections. The minimum green is set as 10 seconds for all cases. The unsignalized intersections are set as follows: no control (62 intersections), yield sign control (24 intersections), and stop sign control (31 intersections). No signal coordination scenarios are considered 


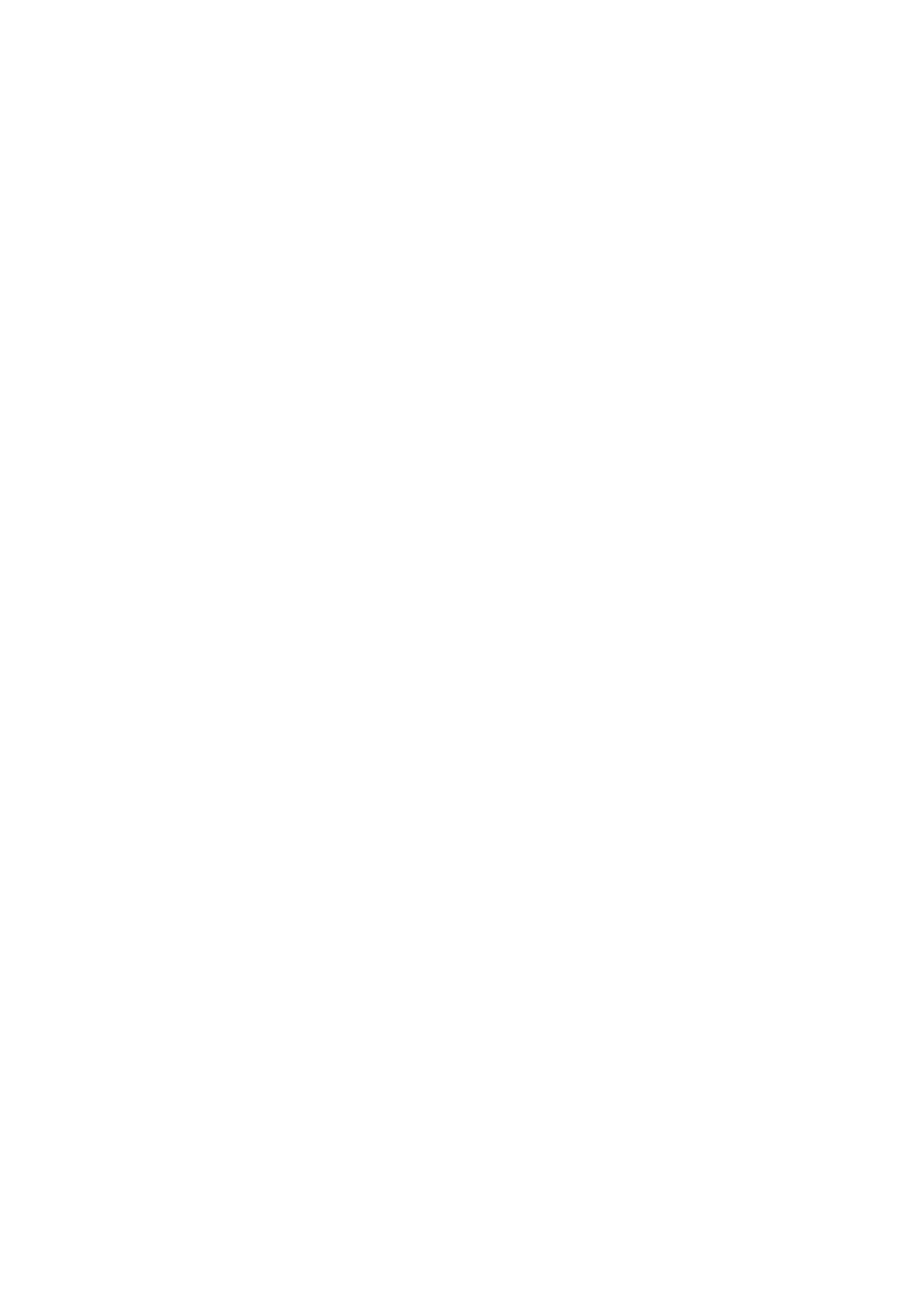

Figure 5. Test Network Showing Simulated Transit Lines 
in these experiments. However, we strongly believe that studying bus priority in coordinated signal system is a logical extension for the current research. Twelve hypothetical bus lines, presented as bold lines in Figure 5, are assumed to connect the main attractions in the network through the main corridors.

Travelers are assumed to have pretrip information on available alternatives. They are also assumed to evaluate these different alternatives according to a prespecified deterministic generalized cost function. Two main trip attributes are considered in these experiments: total travel time and total travel cost. Trip travel time is estimated based on the time-varying network conditions, while trip cost is assumed to be fixed. A travel cost of $\$ 0.20$ per link (which could also vary per link) is assumed across all private car users. A fixed value of time across all travelers taken as $\$ 6.0$ per hour, is used to calculate the generalized cost measure. Of course, a distribution of these values could readily be used instead. A flat bus fare of $\$ 0.50$ is assumed for the 12 bus lines considered. All travelers are assumed to own a car, and to consider transit and intermodal trips that involve at most one transfer along the trip. Thus, four modal options are assumed to be available for each individual: private car, one bus line, two bus lines with one connecting transfer, and park-and-ride with one intermodal transfer. In all experiments, the average vehicle travel and stop times, and the average bus travel and stop times are recorded.

Four experimental factors are considered in this study. One of these factors pertains to the bus priority strategy applied at the signalized intersections: (1) phase extension, (2) red truncation, and (3) phase advance. This factor reflects different levels of bus priority over the automobiles. As described earlier, in the phase extension strategy, the right-of-way is guaranteed only if the bus arrives during the phase that serves this particular approach. In the red truncation and phase advance strategies, the green is advanced to serve the detected bus earlier. The second factor considers two different traffic assignment scenarios: single-mode assignment and intermodal assignment. In the single-mode scenario, buses are simulated only as background traffic. All travelers are assumed to use private cars regardless of the improvements in bus service due bus priority. In the intermodal scenario, travelers are assumed to evaluate the bus option and select it only if it dominates the private car option (according to the mode choice behavior rule). The capability to consider such mode shifts in response to transit operational improvements is one of the contributions of the methodology presented in this article. The third and fourth experimental factors reflect different network congestion levels and different bus intensities in the network, respectively. Three demand 
levels are considered, corresponding to about 13,500, 9,500 and 6,500 vehicles, respectively, over a 20-minute peak loading period. The first demand level represents high traffic congestion conditions, while the last demand level represents light congestion conditions, with the middle level reflecting mild congestion. For the fourth factor, two bus frequency levels are tested which are 12 and 24 buses per hour, respectively. The 12 bus lines shown in Figure 5 are assumed to operate with the same frequency in each experiment.

\section{Results and Analysis}

Table 1 presents a comparison of network performance across the three bus priority strategies, under the single-mode assignment scenario. Each bus priority strategy is evaluated through a separate run of the simulation-assignment model. Furthermore, each of these scenarios is compared against the do-nothing scenario, where no bus priority is considered. In these four experiments, a high bus frequency is considered at a rate of 24 per hour per line. Table 2 presents the same setting as Table 1, however, it considers lower bus frequency at a rate of 12 per hour per line. In the single-mode assignment scenario, buses are simulated only as background traffic and are not considered as a travel mode option. Therefore, the total number of simulated vehicles remains the same, and the effect of priority is primarily in terms of its traffic operational impacts. The average vehicle travel and stop times, together with the average bus travel and stop times are recorded for the three different demand levels. These performance measures are compared with the do-nothing no-bus-priority case (base case).

Regarding savings in the average bus travel and stop times, the two priority strategies (red truncation and phase advance) in which the green is advanced in favor of the detected buses outperform the phase extension strategy. For example, for the highest demand case in Table 1, percentage savings of about 17 percent and 52 percent in the average bus stop time are recorded for the red truncation and the phase advance strategies, respectively. These savings are in turn reflected in average bus travel time savings of 7 percent and 22 percent under the red truncation and the phase advance strategies, respectively. The phase advance strategy, which provides immediate green for any detected bus, outperforms the red truncation strategy in which any detected bus must wait for the other phases in the cycle to receive their minimum green. In all experiments, the effect of the phase extension strategy on the savings in the average bus travel and stop times appears to be minimal. 
Table 1. Comparison of Different Bus Priority Strategies Considering Single-Mode Assignment Where Bus Frequency = 24 Bus/Hour

Table 2. Comparison of Different Bus Priority Strategies Considering Single-Mode Assignment Where Bus Frequency = 12 Bus/Hour 
According to this set of simulation experiments, the corresponding deterioration in the average vehicle travel time, after introducing bus priority, is low. The maximum observed increase in the average vehicle travel time under the single-mode assignment scenario is less than one minute $(\approx 4 \%)$. In some instances, overall savings in the average vehicle travel time were obtained. For example, in Table 1, for the mild congestion level, a one-minute reduction $(\approx 5 \%)$ in average vehicle travel time is recorded for the phase advance strategy. The reason for this apparent "win-win" improvement lies in drivers adjusting their travel paths. Major changes in signal timing at the network level may induce travelers to change paths to take advantage of lower travel times per costs (Abdelfatah and Mahmassani 1998). For instance, the gained day-to-day travel experience, and/or receiving information on traffic congestion, could allow travelers to modify their paths to take advantage of the savings in travel times per costs. This behavioral phenomenon is explicitly modeled in this set of experiments. Travelers are assumed to select the best path for their trips after the change to signal timing with bus priority. A traveler could therefore completely avoid passing through an intersection with bus priority if considerable delay is to be encountered. Incorporating the effect of traveler redistribution in the network in response to major operational changes is an essential capability of the present methodology for the network-level evaluation of traffic operational measures.

Tables 3 and 4 provide a basis for comparing the effect of bus priority on network performance under the intermodal assignment scenario. The phase advance strategy, which is shown to provide the most savings in the bus travel times, is used in this set of experiments. Table 3 shows the results for the high bus frequency rate (24/hour/line), while Table 4 shows the results for the low frequency rate (12/hour/line). In this scenario, bus transit is considered an option in the mode choice set of each traveler. Travelers can therefore switch to transit if this option becomes preferable to private car in light of improvements brought about by the priority strategies. As such, the results of this set of experiments could be interpreted as long-term benefits associated with providing bus priority at signalized intersection. For example, in the highest congestion case in Table 3, a savings of about 24 percent is observed in the average bus travel time after introducing the phase advance bus priority strategy. This savings in average bus travel time results in about 0.4 percent decrease of private car share, 2.9 percent increase in bus share, and more than one minute ( $4.5 \%)$ savings in average vehicle travel time. Similarly, in the highest demand level in Table 4, a savings of 21 percent in average bus travel time reduces private car share by 1.45 percent and increases transit share by about 
Table 3. Comparison of Different Bus Priority Strategies Considering Intermodal Assignment Where Bus Frequency = 24 Bus/Hour

Table 4. Comparison of Different Bus Priority Strategies Considering Intermodal Assignment Where Bus Frequency $=12$ Bus/Hour 
6.57 percent (including park-and-ride), resulting in 3 percent savings in average vehicle travel time.

These results highlight the importance of considering potential modal shifts and reassignment in evaluating traffic network performance under different control schemes, especially when these schemes are expected to affect the modal split in the network (such as bus priority). The results obtained here are intended for illustration purposes only to highlight the methodological approach and its capabilities. The percentage decrease in auto traffic and associated savings in travel time depend mainly on the value of the parameters used in the mode choice function and other input parameters used in the application.

\section{Conclusions}

In this research, three different bus priority strategies are evaluated using a dynamic traffic assignment-simulation framework. These strategies include phase extension, red truncation, and phase advance. A specially modified version of the multimodal assignment-simulation model, DYNASMART, is used in this study. The model dynamically assigns travelers to the different modes and routes according to the prevailing traffic conditions. A set of simulation experiments is performed to compare these bus priority strategies considering two different assignment scenarios: single-mode assignment and intermodal assignment. The results of these experiments show that the phase advance and red truncation strategies outperform the phase extension strategy. In contrast to previous studies, when correctly modeling the vehicles reassignment phenomenon, the deterioration in the average vehicle travel time was minor. Under the intermodal assignment scenario, the savings in average bus travel time could potentially attract more travelers to use the bus instead of private cars. This reduces the congestion level in the network as indicated by the reduction in average vehicle travel time. Extension of this work includes testing the model in real-world situations considering different operational scenarios. Another extension is to study bus priority strategies in a coordinated signal system to evaluate its possible disruption effect on the timing of the coordinated signals. 


\section{References}

Abdelfatah, A. S., and H. S. Mahmassani. 1998. System optimal time-dependent path assignment and signal timing in traffic network. Transportation Research Record 1645: 185-193.

Abdelghany, K. 2001. Stochastic dynamic traffic assignment for intermodal transportation networks with consistent information supply strategies. Ph.D. Dissertation. The University of Texas at Austin, Austin, TX.

Abdelghany, K., D. Valdes, A. Abdelfatah, and H. Mahmassani. 1999. Real-time dynamic assignment and path-based signal coordination: Application to network traffic management. Transportation Research Record 1667: 67-76.

Abdelghany, K., and H. S. Mahmassani. 2001. Assignment-simulation model for intermodal transportation networks. Transportation Research Record 1771: $52-60$.

Baker, R., et al. 2002. An overview of transit signal priority handbook. Washington, DC: Intelligent Transportation Society of America.

Bell, M. G. H. 1992. Future directions in traffic signal control. Transportation Research A, 26: 303-313.

Chang, E., and A. Ziliaskopoulos. 2003. Data challenges in development of a regional assignment: Simulation model to evaluate transit signal priority in Chicago. Transportation Research Record 1841: 12-22.

Chang, G-L., M. Vasudevan, and C. Su. 1995. Bus-priority under adaptive signal control environments. Transportation Research Record 1494: 146-154.

Chang, J., J. Collura, F. Dion, and H. Rakha. 2003. Evaluation of service reliability impacts of traffic signal priority strategies for bus transit. Final Report. National ITS Implementation Research Center. Virginia Tech Transportation Institute.

Chard, B. M., and C. J. Lines. 1987. TRANSYT: The latest developments. Traffic Engineering and Control 28: 387-390.

Garrow, M., and R. Machemehl. 1997. Development and evaluation of transit signal priority strategies. Report SWUTC/97/472840-00068-1. Austin, TX: Center for Transportation Research. 
Hu, Ta-Yin. 1995. Dynamic analysis of network flows under advanced information and control systems. Ph.D. Dissertation. The University of Texas at Austin, Austin, TX.

Heydecker, B. 1983. Capacity at a signal-controlled junction where there is priority for buses. Transportation Research B, 17: 341-357.

Jayakrishnan, R., H. S. Mahmassani, and T.-Y. Hu. 1994. An evaluation tool for advanced traffic information and management systems in urban network. Transportation Research C, 2: 129-147.

Khasnabis, S., R. R. Karnati, and R. K. Rudra Raju. 1996. A NETSIM based approach to evaluate bus priority strategies. Transportation Research Record 1554: 80-89.

Longfoot, J. E. 1982. SCATS-Development of management and operation systems. International Conference on Road Traffic Signalling, London, United Kingdom.

Lowrie, P. R. 1982. The Sydney coordinated adaptive traffic system-Principles, methodology, algorithms. N.S.W., Australia: Department of Main Roads.

Mahmassani, H. S., et al. 2000. DYNASMART-P: Intelligent transportation network planning tool. Austin, TX: Center for Transportation Research, The University of Texas at Austin.

Mirchandani, P., and D. Lucas. 2004. Integrated transit priority and rail/emergency priority in real-time traffic adaptive signal control. Intelligent Transportation Systems Journal 8: 101-115.

Nash, A. 2003. Implementing Zurich's transit priority program. Transportation Research Record 1835: 59-65.

Radwan, A. E., and D. A. Benevell. 1983. Bus priority strategy: Justification and environmental aspects. Journal of Transportation Engineering 109: 88-106.

Sims, A. G., and A. B. Finlay. 1984. SCATS, splits and offsets simplified (S.O.S.). ARRB Proceedings 12, Part 4.

Smith, H., B. Hemily, and M. Ivanovic. 2005. Transit signal priority (TSP): A planning and implementation handbook. Washington, DC: Intelligent Transportation Society of America. 
Sunkari, S. R., T. U. Beasley, and D. B. Fambro. 1995. Model to evaluate the impacts of bus priority on signalized intersections. Transportation Research Record 1494: 117-123.

Yagar, S. 1993. Efficient transit priority at intersections. Transportation Research Record 1390: 10-15.

\section{About the Authors}

Khaled Abdelghany (khaled@engr.smu.edu) received a Ph.D. in civil engineering from the University of Texas Austin in 2001, and B.Sc. and M.Sc. from Cairo University in 1992 and 1996, respectively. He is currently an environmental and civil engineering assistant professor at Southern Methodist University, Dallas. Prior to joining SMU, Dr. Abdelghany worked in the operations planning group in the Information Services Division at United Airlines. His research interests include modeling intermodal transportation networks, intelligent transportation systems, and airlines operation management. Dr. Abdelghany been has published in several journals, including Transportation Research Record, Annals of Operations Research, and Journal of Air Transport Management. He is a member of American Society of Civil Engineers, Institute of Transportation Engineers, and Institute for Operations Research and the Management Sciences.

Ahmed Abdelghany (Abdel776@erau.edu) is an assistant professor at the College of Business, Embry-Riddle Aeronautical University. Prior to his association with Embry-Riddle, Dr. Abdelghany was an assistant professor in the Department of Civil and Environmental Engineering at the University of Alaska, Fairbanks. Also, he worked for several years as a senior analyst in the Information Services Division of United Airlines. Dr. Abdelghany holds a doctorate in civil engineering from the University of Texas at Austin (2001). His research in the areas of transportation planning, traffic engineering, and airline operation management have appeared in several journals, including Transportation Research Record, Annals of Operations Research, Air Transportation Management, and Air Traffic Control Quarterly.

HaNı S. MAhmassanı (masmah@mail.umd.edu) received a Ph.D. in transportation systems from the Massachusetts Institute of Technology in 1982, an M.S. in transportation engineering from Purdue University in 1978, and a B.S. in civil engineering from the University of Houston in 1976. He is the first holder of the Charles Irish Sr. Chair in Civil and Environmental Engineering at the University of Maryland, where he is also the founding director of the Maryland Transportation 
Initiative. He joined the University of Maryland in August 2002, after 20 years on the faculty at the University of Texas at Austin, where he was most recently the A. Abou-Ayyash Centennial Professor in transportation engineering, professor of management science and information systems, and director of the Advanced Institute for Transportation Infrastructure Engineering and Management. He specializes in multimodal transportation systems analysis, planning and operations, dynamic network modeling and optimization, transit network planning and design, dynamics of user behavior and telematics, telecommunication-transportation interactions, large-scale human infrastructure systems, and real-time operation of logistics and distribution systems.

AKmal Abdelfatah (akmal@aus.ac.ae) is an assistant professor of civil engineering at the American University of Sharjah, United Arab Emirates. He has a Ph.D. from the University of Texas at Austin and has worked as a transportation planning and traffic engineering consultant/researcher on many projects in the Middle East and the United States. Dr. Abdelfatah's research interests are focused on dynamic traffic assignment, traffic operations, and ITS applications. 